\title{
Perioperative chemoimmunotherapy in a patient with stage IIIB non-small cell lung cancer
}

\author{
Hengrui Liang ${ }^{1 \#}$, Hongsheng Deng ${ }^{1 \#}$, Wenhua Liang ${ }^{1 \#}$, Kai Guo ${ }^{2}$, Ziqing Gao ${ }^{2}$, Ory Wiesel ${ }^{3}$, \\ Raja M. Flores ${ }^{4}$, Kimberly Song ${ }^{4}$, Bassam Redwan ${ }^{5}$, Marcello Migliore ${ }^{6}$, Shuben Li ${ }^{1}$, Weiqiang Yin ${ }^{1}$, \\ Jianxing $\mathrm{He}^{1}$
}

${ }^{1}$ Department of Thoracic Surgery and Oncology, the First Affiliated Hospital of Guangzhou Medical University, State Key Laboratory of Respiratory Disease \& National Clinical Research Center for Respiratory Disease, Guangzhou 510120, China; ${ }^{2}$ Guangzhou Medical University, Guangzhou 510120, China; ${ }^{3}$ Division of Thoracic Surgery, Department of Surgery, Maimonides Medical Center, Brooklyn, NY, USA; ${ }^{4}$ Department of Thoracic Surgery, Mount Sinai Health System, Icahn School of Medicine at Mount Sinai, New York, NY, USA; ${ }^{5}$ Department of Thoracic Surgery, Klinik am Park, Klinikum Westfalen, Lünen, Germany; ${ }^{6}$ Section of Thoracic Surgery, Department of Surgery and Medical Specialities, Policlinico University Hospital, University of Catania, Italy

\#These authors contributed equally to this work.

Correspondence to: Shuben Li; Weiqiang Yin; Jianxing He. Department of Thoracic Surgery and Oncology, The First Affiliated Hospital of Guangzhou Medical University, State Key Laboratory of Respiratory Diseases, National Clinical Research Center for Respiratory Disease, Guangzhou 510120, China. Email: 13500030280@163.com; yinweiqiang88@163.com; drjianxing.he@gmail.com.

\begin{abstract}
We present a case of a 56-year-old male patient with stage IIIB (T3N2M0) poorly differentiated squamous cell carcinoma of the lung. Four cycles of chemotherapy were first applied, and the patient had stable disease. However, the patient refused to receive radiotherapy, therefore second-line treatment chemotherapy combined with anti-PD-1 immunotherapy was applied. Partial response was reached at the $4^{\text {th }}$ cycle of chemotherapy combined with anti-PD-1 immunotherapy. The neoadjuvant strategy was prolonged to 10 cycles but no significant change was observed on tumor size. The patient then underwent videoassisted thoracoscopic left lower lobectomy. Eight cycles of adjuvant PD-1 immunotherapy were applied postoperatively. Perioperative immunotherapy demonstrated good curative effect in this patient and no recurrence was observed at the clinic 40 months following surgery. Here we intend to explore the concept of immunotherapy combined with chemotherapy and surgery in neoadjuvant and adjuvant setting, and to investigate the possibility of extending this strategy in patients with stage IIIB non-small cell lung cancer (NSCLC).
\end{abstract}

Keywords: Non-small cell lung cancer (NSCLC); perioperative PD-1 inhibitor; stage IIIB

Submitted Jan 4, 2020. Accepted for publication Jan 26, 2020.

doi: 10.21037/atm.2020.01.118

View this article at: http://dx.doi.org/10.21037/atm.2020.01.118

\section{Introduction}

Checkpoint inhibitors such as programmed cell death protein 1 inhibitors (PD-1 inhibitors) represent an essential part of tumor immunotherapy and have attracted much attention over the last years. In 2012, the CA209-003 study confirmed that Nivolumab has antitumor activity in nonsmall cell lung cancer (NSCLC) for the first time (1). After a short period of time in 2015, PD-1/PD-L1 immune checkpoint inhibitors (ICIs) represented by Nivolumab were proven to be far more effective than docetaxel in second-line treatment of NSCLC (2). Later in 2017 , pembrolizumab was first approved to be used as a first-line treatment for EGFR/ALK/ROS1 negative NSCLC patients with PD-L1 expression $\geq 50 \%$ (KEYNOTE 024), which became a milestone and represented the new era of PD-1 immunotherapy (3).

The current case represents a case of a male patient 


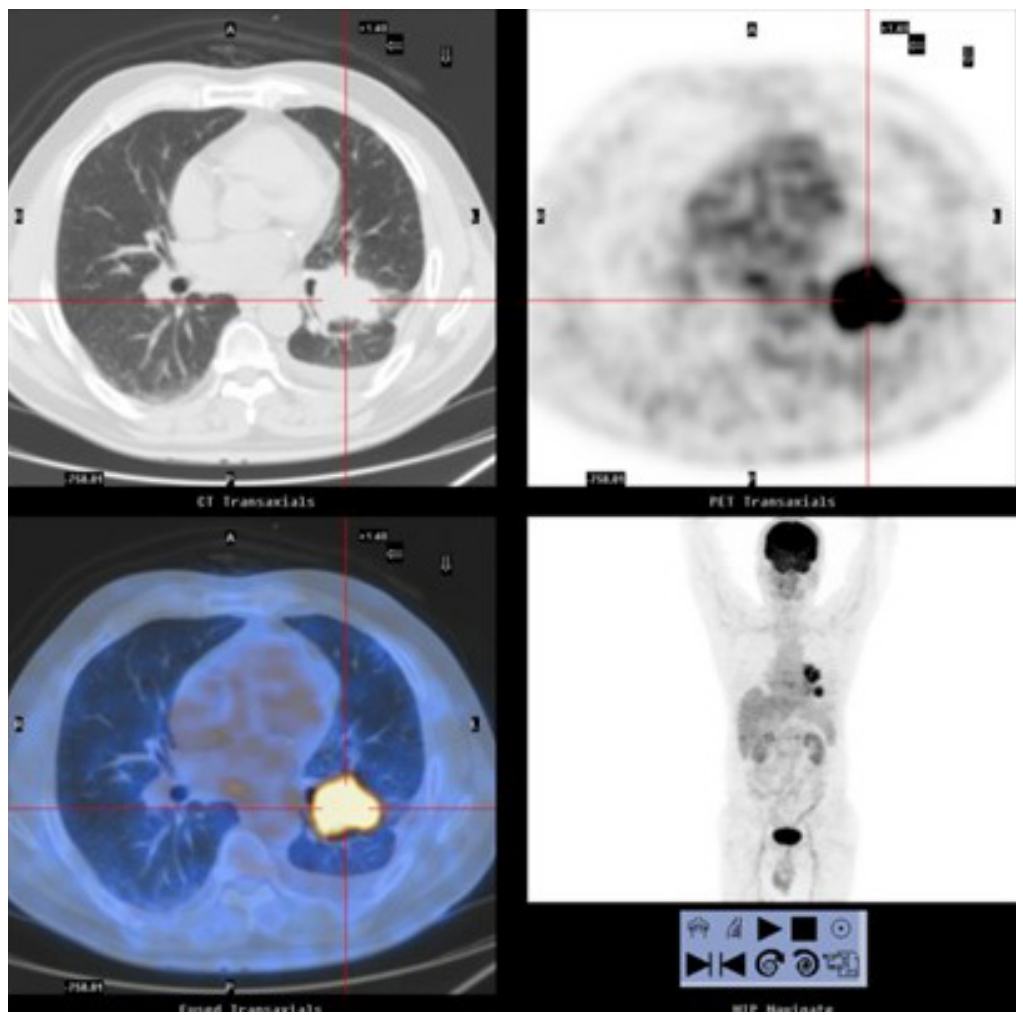

Figure 1 PET-CT examination proving multiple lung cancers occurred in the posterolateral basal segment of the left lower lobe (4.5 $\mathrm{cm}$ $\times 3.7 \mathrm{~cm}$; SUV 10.9) and the root of the left pulmonary hilum (4.0 cm × $2.9 \mathrm{~cm}$; SUV 10.4) (Baseline) (2014-09-17).

with stage IIIB (T3N2M0) poorly differentiated squamous cell carcinoma of the lung. Chemotherapy combined with PD-1 immunotherapy was first applied, followed by videoassisted thoracoscopic surgery (VATS) left lower lobectomy and postoperative adjuvant PD-1 immunotherapy. Herein, we attempt to discuss the treatment strategy for the IIIB (T3N2M0) NSCLC patient in the era of immunotherapy.

\section{Case presentation}

A 56 years old, heavy smoker male was admitted to our hospital for recurrent cough over a year and a half with back pain for the past 10 days. His past medical history was non-contributory. With further questioning, the patient reported that he noted dry, persistent non-productive cough that started 6 months ago. Further, the patient denied constitutional symptoms such as fever, night sweats, chills and palpitations. The patient was referred initially to another hospital and computed tomography scan (CT) was performed showing a left lower lobe lung mass with mediastinal lymph node enlargement. He was started on antibiotics without improvement and was referred to our center.

On September 15, 2014, the patient was admitted to our hospital for further treatment. Patient's past disease history and family history were unremarkable. Nicotine abuse with 60 smoking pack years was present. With the exception of attenuated breathing sounds in the left lower lung on auscultation, physical examination revealed no further abnormalities.

His initial positron-emission-tomography scan (PETCT) (2014-09-17) revealed (Figure 1): two lung masses in the posterolateral basal segment of the left lower lobe $(4.5 \mathrm{~cm} \times 3.7 \mathrm{~cm}$; SUV 10.9) and the root of the left pulmonary hilum $(4.0 \mathrm{~cm} \times 2.9 \mathrm{~cm}$; SUV 10.4), combined with left pulmonary hilum and mediastinal lymph adenopathy (maximum short diameter $1.0 \mathrm{~cm}$; SUV 2.5-3.7), a small amount of pleural effusion on the left side of the thorax. The patient underwent Brain Magnetic Resonance imaging (MRI) which was negative for metastatic disease. Endobronchial Ultrasound staging (EBUS) showed positive $\mathrm{N} 2$ disease, with stations $4 \mathrm{~L}, 7$ and 11L metastasis. 


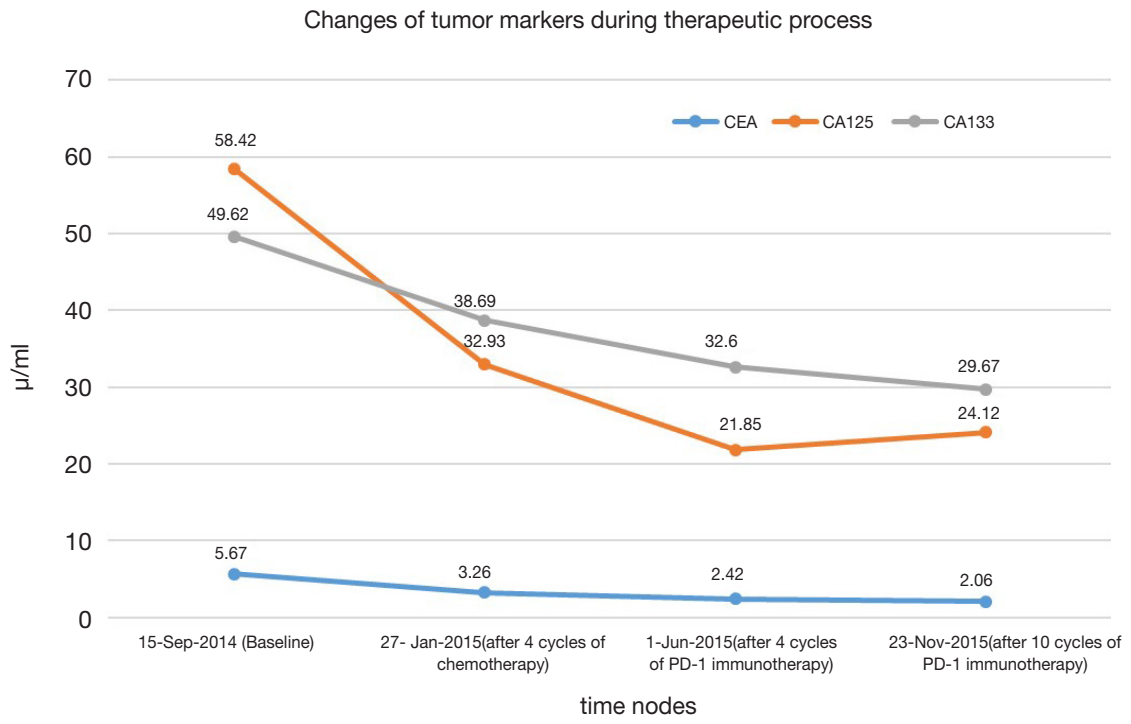

Figure 2 An overview of tumor markers (CEA/CA125/CA133) concentration in this case during the therapeutic process.

Immunohistochemistry of biopsy samples (11L): P63 (+), TTF1 (-), NapsinA (-), CK5/6 (+). Changes of the tissue were consistent with poorly differentiated squamous cell carcinoma. ALK (-), ALK-negative control (-). Genetic test: EGFR mut (-), ALK mut (-). PD-L1 expression: unknown; TMB: unknown. Tumor marker(baseline): CEA: 5.67; CA125: 58.42; CA133: 49.62. Diagnosis: squamous cell carcinoma of the left lower lung (cT3N2M0, stage IIIB).

The patient was presented during our multidisciplinary tumor board and a treatment plan was established starting with induction chemotherapy plus radiotherapy followed by restaging and re-evaluation. The patient underwent 4 cycles of albumin taxol $400 \mathrm{mg}$ D1+ carboplatin $240 \mathrm{mg}$ D1 and 300 mg D2. Tumor markers (CEA/CA125/CA133) were respectively measured during each cycle of chemotherapy, and each value gradually declined (Figure 2). Repeated PETCT after completion of the 4th cycle (2015-01-27) (Figure 3) showed response both in the posterolateral basal segment of the left lower lobe $(3.9 \mathrm{~cm} \times 3.3 \mathrm{~cm} v s .4 .5 \mathrm{~cm} \times 3.7 \mathrm{~cm})$ and in the root of the left pulmonary hilum $(3.7 \mathrm{~cm} \times 2.9 \mathrm{~cm} v s$. $4.0 \mathrm{~cm} \times 2.9 \mathrm{~cm}$ ) compared to prior scan. Patient's condition was considered as stable disease (SD). Unfortunately, the patient refused to receive completion radiotherapy at this point, hence immunotherapy was proposed. PD-1 inhibitor (pembrolizumab) was used [150mg; q4w; for 10 cycles] from February 16, 2015 to November 23, 2015. Chest CT following the 4th cycle of immunotherapy (2015-06-01) (Figure 4) demonstrated durable response in the mass size at the posterolateral basal segment of the left lower lobe $(2.8 \mathrm{~cm} \times 2.2 \mathrm{~cm} v s .4 .0 \mathrm{~cm} \times 4.2 \mathrm{~cm})$ and the root of the left pulmonary hilum $(2.6 \mathrm{~cm} \times 2.3 \mathrm{~cm} v s .3 .5 \mathrm{~cm} \times 3.5 \mathrm{~cm})$. Moreover, the size of the metastatic left pulmonary hilum and mediastinal lymph nodes declined as well. Given the patient's response we elected to continue the second-line treatment with PD-1 immunotherapy. PET-CT scan was performed after the 10th cycle of immunotherapy (2015-1123) (Figure 5) and demonstrated continued response in the posterolateral basal segment mass $(2.3 \mathrm{~cm} \times 2.4 \mathrm{~cm}$; SUV 2.9) and root of the left pulmonary hilum $(3.1 \mathrm{~cm} \times 2.3 \mathrm{~cm})$. Of the left pulmonary hilum, the mediastinal lymph adenopathy showed little change compared with previous images with stable minimal size and stable FDG uptake (original SUV 2.2-2.7). Tumor markers following the 10th cycle of immunotherapy demonstrated a significant reduction compared to these withdrawn following the $4^{\text {th }}$ cycle of immunotherapy (Figure 2).

Given his significant durable response in the face of loco regional disease and without distant metastases, the patient was presented again in our multidisciplinary tumor board and was found eligible for surgical exploration with intent for cure. On December $23^{\text {rd }}, 2015$ the patient underwent thoracoscopic exploration and left lower lobectomy with mediastinal lymph node dissection. A $2.5 \mathrm{~cm}$ residual tumor was seen in the posterolateral basal segment of the left lower lobe, together with a $2 \mathrm{~cm}$ lymph node at the pulmonary hilum. Desmoplastic reaction (local pleural adhesion, thickened pleura) and a small amount of pleural 


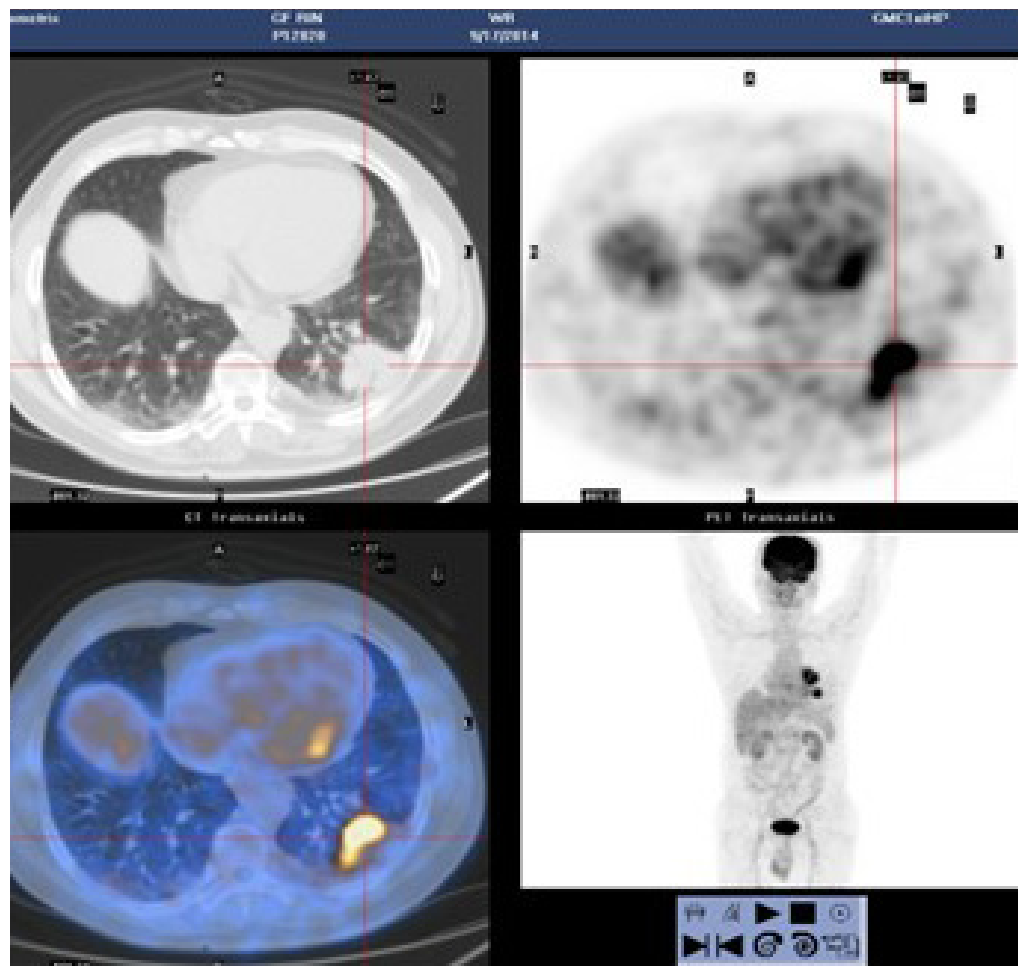

Figure 3 PET-CT examination after 4 cycles of chemotherapy demonstrating reduction of lesions (2015-01-27).

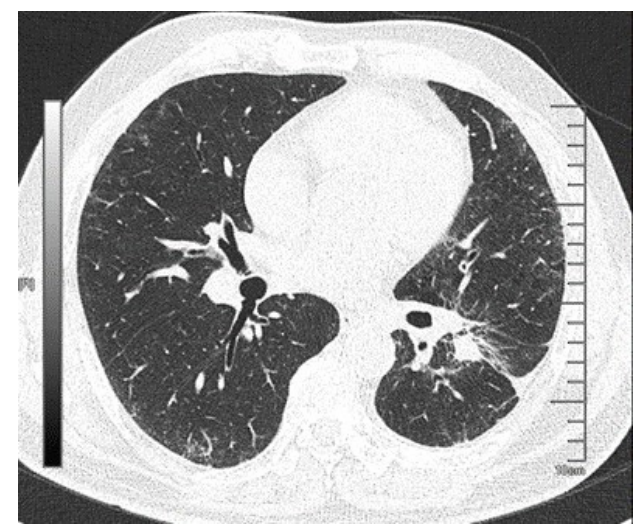

Figure 4 CT scan after 4 cycles of PD-1 immunotherapy demonstrating the therapeutical effect as PR (2015-06-01). PR, pathological response.

effusion was found (negative cytology) as well. Due to the chemo- and immunotherapy induced tissue alteration with partially massive adhesions and significantly thickened pleura, intraoperative blood loss was $1,000 \mathrm{~mL}$. The patient was transfused 4 units of packed red blood cells and 1 unit of fresh frozen plasma intraoperatively. The patient was

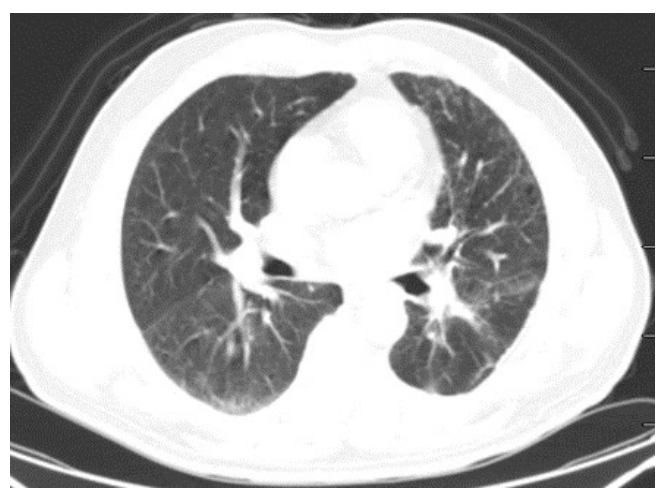

Figure 5 CT scan after 10 cycles of PD-1 immunotherapy demonstrating no significant change compared to that after 4 cycles of PD-1 immunotherapy (2015-11-23).

transferred to ICU after surgery. Following an uneventful postoperative course, the patient was discharged on postoperative day (POD) 6 .

Postoperative pathological examination revealed changes of moderately-poorly differentiated squamous cell carcinoma of the lung after chemotherapy. No tumor invasion was found in the visceral pleura and the surgical 


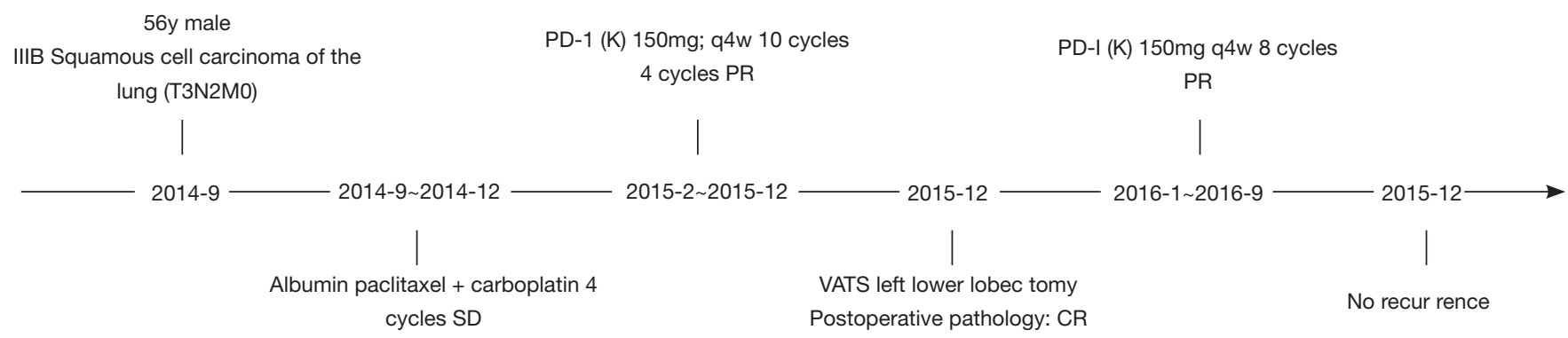

Figure 6 Treatment flow chart of the patient from September 2014 to April 2019.

margins were negative. One para-bronchial lymph node $(0 / 1)$, two lymph nodes of group $4(0 / 2)$, six lymph nodes of group 7 (0/6), and eight lymph nodes of group 11 $(0 / 8)$ were examined, none of which showed tumor tissue. Immunohistochemistry examination: TTF1 (-); CK7 (-); about (+); CK5/6 (+); P40 (+). Pathological response rate: unknown; PD-L1 expression: unknown. Postoperative staging: T1cN0M0 (stage IA).

The patient continued to receive the postoperative adjuvant immunotherapy, with the Pembrolizumab 150mg; $\mathrm{q} 4 \mathrm{w}$; for 8 cycles. Asymptomatic interstitial pneumonia was diagnosed a year after surgery, treated medically. The patient was last seen at the clinic 40 months following surgery (March 28th, 2019), no recurrence was observed (Figure 6).

\section{iMDT discussion}

Discussion among oncologists and thoracic surgeons from the First Affiliated Hospital of Guangzhou Medical University

Lung cancer is the leading cause of cancer-related deaths worldwide, with approximately 1.8 million people dying of lung cancer each year worldwide. In China, lung cancer is the most commonly diagnosed cancer in men and the second most commonly diagnosed cancer in women. In 2015 , there was an estimate of 733,300 new lung cancer diagnoses and 610,200 deaths of lung cancer (4). The incidence of NSCLC accounts for about $85 \%$ of total lung cancers. According to the 2019 NCCN guidelines of NSCLC, standard treatment for stage I/II/partial IIIa NSCLC is radical resection (3). However, most patients have advanced stages at the time of primary diagnosis (stage IIIb or above) (5). Single-agent PD-1/L1 inhibitors and combinations of $\mathrm{PD}-1 / \mathrm{L} 1$ inhibitors with chemotherapy or other ICIs like CTLA-4 have been explored as frontline treatments for advanced NSCLC patients (6). The patient discussed in this case report was first diagnosed in 2014, stage IIIB (cT3N2M0), and not suitable for primary surgical resection. According to the $2019 \mathrm{NCCN}$ guidelines, "concurrent chemoradiotherapy + PD-1 maintenance therapy" is recommended for patients with IIIB (T3N2M0) NSCLC $(3,7)$. Also, the expression of PDL1 was a routine examination in our center. However, since this case was first diagnosed in 2014, the examination of PD-L1 expression was not routinely conducted. The 2014 version of the NCCN guidelines recommended that the standard treatment of stage IIIB (T3N2M0) NSCLC was induction chemotherapy \pm radiotherapy, and surgery should be considered later if no obvious progression was found. However, the patient refused to receive radiotherapy after chemotherapy, so a more radical and suitable for earlystage lung cancer therapy was elected, and "sequential chemotherapy and PD-1 immunotherapy, plus surgery and postoperative PD-1 maintenance treatment", was adopted.

In this case report, there are several issues with the preoperative strategy that can be further investigated:

(I) For stage IIIB cT3N2M0 NSCLC patients with EGFR mut (-) and ALK mut (-), which is better among single-agent PD-1 inhibitor, single-agent chemotherapy, or chemotherapy combined with PD-1 immunotherapy? The KEYNOTE-024 study has already indicated that overall survival (OS) for patients treated with chemotherapy combined with PD-1 immunotherapy was higher than that of single chemotherapy $(80.2 \%$ vs. $72.4 \%$, HR $0.6 ; 95 \%$ CI: $0.41-0.89, \mathrm{P}=0.005)$ (8). Also, an indirect comparison of treatment measures (ITC) analysis suggested that in the subgroup with low expression of PD-L1 <1\%, PD-1/PD-L1 combined with chemotherapy was superior to chemotherapy alone in OS (HR 0.78, 0.67-0.90) and PFS (progression-free 
survival) (HR 0.72, 0.65-0.80). In the subgroup with high expression of PD-L1 $\geq 50 \%$, the OS (HR 0.61, 0.49-0.77) and PFS (HR 0.41, 0.34-0.49) of PD-1/PD-L1 combined with chemotherapy were longer than those of chemotherapy alone, and the PFS of PD-1/PD-L1 combined with chemotherapy was also better than that of the singleagent PD-1/PD-L1 group (HR 0.71, 0.51-0.99) (6). The result showed that PD-1/PD-L1, combined with chemotherapy, is superior to single-agent chemotherapy or chemotherapy alone, regardless of the high or low expression of PD-1/PD-L1. Therefore, the combination of chemotherapy and PD-1 immunotherapy was a better option for this patient. However, attention should also be paid to toxic side effects of combination therapy, for the reason that the incidence of treatment-related and immunemediated adverse events is the highest in the combination therapy group (6).

(II) If the combination therapy is chosen, what is the sequence of using chemotherapy and PD-1 immunotherapy? Which is better, sequential or concurrent chemotherapy and PD-1 immunotherapy? Related studies have suggested that chemotherapy upregulates the absolute number and function of tumor-infiltrating lymphocytes (9). Also, drugs that are used in chemotherapy can increase the expression of NF- $\kappa B$ in activated B cells and CD8+ T cells (10), as well as inducing the proliferation of CD8+ T cells. These studies provide a theoretical basis for chemotherapy function of "heating tumors from cold," meaning that chemotherapy can change the tumor microenvironment from "desert" to "inflamed one." (11) Sequential chemotherapy and immunotherapy may, therefore, be more rational in this case.

(III) How long should preoperative PD-1 adjuvant therapy be used? The CheckMate 159 clinical study included 21 newly diagnosed, untreated resectable stage I/II/IIIa (tumor volume $>2 \mathrm{~cm}$ ) patients with NSCLC. Nivolumab ( $3 \mathrm{mg} / \mathrm{kg}$ ) was given to these patients preoperatively (q2w; 2 cycles). The tumor was surgically resected 4 weeks after the first dose of Nivolumab. Followup results after 30 days: Imaging response: $2 / 21$ (10\%) PR; 18/21 (85\%) SD; $1 / 21$ (5\%) PD. Pathological response (PR): 9/20 (45\%) major pathologic response (MPR); 3/20 (15\%) complete pathologic response (12). The CheckMate 159 clinical study indicated that it might be appropriate to use 2 cycles of PD-1 adjuvant therapy. In this case, there was no significant change from the 4th to the 10th cycle of PD-1 immunotherapy according to the CT scans (1 cycle: false progress; 4 cycles: PR; 10 cycles: PR), so the suitable time for operation may be brought forward to the 4th cycle of immunotherapy, when the patient's condition reached PR. It is feasible to bring forward the surgery time to the 2 nd to 3 rd cycles of immunotherapy, according to the results of the CheckMate159 clinical study. However, in this case, PD-1 immunotherapy was performed in 2014, the actual dose was less than the standard dose recommended by the current NCCN guidelines for NSCLC $(150 \mathrm{mg}$; q4w vs. $200 \mathrm{mg}$; $\mathrm{q} 3 \mathrm{w}$ ) (3). Therefore, careful consideration should be given to the calculation of the cycles of immunotherapy.

(IV) In this case, the patient achieved PR after 10 cycles of PD-1 immunotherapy. Which was the next intervention measure: surgery, radiotherapy, or systemic maintenance therapy? If the choice was surgery, when was the proper time? According to the 2019 version of NCCN guidelines for NSCLC, the standard treatment for IIIB (T3N2M0) patients was induction chemotherapy \pm radiotherapy. If the disease has no significant progress, surgery \pm chemotherapy \pm radiotherapy were recommended (3). Furthermore, the appropriate time for surgery should follow the individualized strategy, whether conforming to the indication of surgical resection or not should be based on the surgeon's judgment.

This case report discusses the significant effect of immunotherapy on the patient, which might indicate that the patient was of high expression of PD-L1. Several publications have already shown that high expression of PD-L1 can be significantly increased after targeted therapy and chemotherapy (13), which benefits lung cancer patients. In our case, sequential chemotherapy combined with PD-1 treatment + surgery + PD-1 maintenance in a patient with stage IIIb NSCLC led to a good clinical result. Further studies should examine this concept in larger patient cohorts.

Several issues regarding the immunotherapy strategy were further discussed as follows:

Question 1: What is the necessity of postoperative adjuvant therapy? If major pathological remission or complete pathological remission is achieved, is postoperative adjuvant therapy unnecessary? Expert opinion 1: Dr. Bassam Redwan

The impact of postoperative adjuvant therapy for patients with resected NSCLC has been investigated in several clinical trials (14). The Lung Adjuvant Cisplatin Evaluation (LACE) meta-analysis included 4,584 patients from five randomized trials demonstrated a benefit of adjuvant cisplatin-based chemotherapy with a hazard ratio of 0.89 
and a 5 -year survival rate of 5.3\% (15). Current standard of therapy is a cisplatin-based doublet chemotherapy after surgical resection in patients with pathologic tumor stages II and III (according to the eighth edition of the TNM classification). Patients with tumors greater than $4 \mathrm{~cm}$ showed a benefit after adjuvant chemotherapy (16). Due to the presence of micro-metastases at time of surgery, which might lead to systematic relapses in further course of disease, adjuvant therapy might improve OS and diseasefree survival (DFS) by eliminating such metastases. In advanced tumor stages, such as in our case, presence of micro-metastases is more likely, and therefore, adjuvant therapy would be recommended, even if complete pathological remission of the primary tumor is achieved.

\section{Expert opinion 2: Dr. Marcello Migliore}

Although there is no strong scientific evidence I think that postoperative adjuvant therapy could be necessary even when complete pathological remission is achieved because the tumor was advanced at the initial evaluation.

\section{Expert opinion 3: Dr. Ory Wiesel}

Although he completed neo-adjuvant chemotherapy course, given the fact that he had aggressive and advanced tumor and the fact that he refused radiation therapy-the patient might benefit from postoperative chemotherapy even though there is no good scientific evidence of its efficacy.

Question 2: Compared with the 12 cycles of adjuvant therapy recommended by the "Pacific model," only 8 cycles of PD-1 immunotherapy were used in this case after surgery. How to consider the duration of adjuvant therapy in clinical practice?

\section{Expert opinion 1: Dr. Bassam Redwan}

Duration of adjuvant therapy should always be individually adapted to the current clinical situation and the drug-related adverse events occurring during the course of therapy. In the "PACIFIC" trial, Grade 3 or 4 adverse events occurred in $29.9 \%$ of the patients receiving durvalumab and a total of $15.4 \%$ of patients discontinued the study drug due to adverse events (7).

\section{Expert opinion 2: Dr. Marcello Migliore}

In clinical practice, the only way to decide the duration of adjuvant immunotherapy is to perform a randomized controlled trial (RCT).

Expert opinion 3: Dr. Raja M. Flores \& Dr. Kimberly $\mathcal{F}$. Song

There are as yet no set guidelines regarding the exact duration or timing of adjuvant immunotherapy. Current ongoing trials are investigating the role of immunotherapy after adjuvant chemotherapy with and without radiation, as well as in completely resected NSCLC (17).

\section{Expert Opinion 4: Dr. Ory Wiesel}

The PACIFIC trial is not applicable to this specific patient. Although it is reasonable to add immunotherapy in advanced disease postoperatively, there is no available published data to guide us on the timing nor duration of adjuvant immunotherapy applicable to patient population such as the patient presented. There are ongoing trials evaluating this specific question that should be published in the near future.

Question 3: Should postoperative PD-1 adjuvant therapy be combined with chemotherapy? Expert opinion 1: Dr. Bassam Redwan

In a recent meta-analysis, 13 clinical trials investigating the impact of different ICIs plus conventional treatment in advanced lung cancer patients were included (18). The pooled results demonstrated that combination strategy based on ICI significantly improved progressionfree survival $(\mathrm{PFS})(\mathrm{HR}=0.66, \mathrm{P}<0.001)$ and $\mathrm{OS}(\mathrm{HR}$ $=0.77, \mathrm{P}<0.001)$ in overall population. Hereby, greatest PFS improvement was seen in the group of PD- 1 based combination $(\mathrm{HR}=0.54, \mathrm{P}<0.001)$. In a recent study by Lacour et al, patients receiving adjuvant chemotherapy showed significant upregulation in PD-L1 expression at recurrence, supporting a combinatorial therapy with chemotherapy and anti-PD(L)1 treatment (19).

\section{Expert opinion 2: Dr. Marcello Migliore}

It is difficult for me also to comment if PD-1 adjuvant therapy should be combined with chemotherapy but certainly the combination of both can improve therapeutic efficacy and safety of cancer immunotherapy, but again a RCT is necessary.

\section{Expert opinion 3: Dr. Ory Wiesel}

There is no clinical data to support this. There are ongoing clinical trials that designed to address this exact question which hopefully will provide some answers in the near future.

Question 4: If the patient recurred after surgery, can PD-1 immunotherapy be continued? Expert opinion 1: Dr. Marcello Migliore

Nevertheless, I would have followed EACTS guidelines to take more nodes in the mediastinum (at least 3 mediastinal stations) to decrease the recurrence. Moreover, further studies are necessary to discover factors which predict single agents or combination therapies that will work best for 
recurrent patients after surgery.

Expert opinion 2: Dr. Raja M. Flores \& Dr. Kimberly $\mathcal{J}$.

Song

In this particular case, there was a radiographically documented reduction in tumor burden despite unknown PD-L1 expression, and the response is likely to have reduced the extent of surgery that would have been required. With this in mind it would be reasonable to consider resuming immunotherapy in case of recurrence presuming the patient did not develop pneumonitis or other therapy-related toxicities.

\section{Expert opinion 3: Dr. Ory Wiesel}

The current data supports adding Immunotherapy to chemotherapy in metastatic disease or non-resectable advanced NSCLC. However, in this particular patientgiven the nice response in the preoperative setting I would favor resuming immunotherapy with or without chemotherapy once he recurs.

Question 5: Does the expression of PD-L1 affect the strategies of adjuvant/neoadjuvant therapy?

Expert opinion 1: Dr. Bassam Redwan

In a recent meta-analysis, patients showed a benefit from combination therapy based on ICI over chemotherapy, regardless of PD-L1 expression (18). The improvement in PFS with combination versus chemotherapy did not differ by PD-L1 expression. Therefore, PD-L1 expression should not affect the treatment strategies of patients with NSCLC. It is therefore necessary to define another biomarker of ICI efficacy.

Tumor mutation burden (TMB) is being currently investigated as a biomarker of ICI efficacy (20). OwadaOzaki et al. conducted a study investigating the prognostic Impact of TMB in patients with completely resected NSCLC (20). TMB greater than 62 was associated with worse OS of patients with NSCLC. The authors concluded that if high TMB is a predictor of the efficacy of ICIs, postoperative adjuvant therapy with ICIs may contribute to improvement of recurrence and OS.

Expert opinion 2: Dr. Marcello Migliore

PD-L1 expression in relation to prognosis both in the adjuvant/ neoadjuvant chemotherapy setting is still controversial, and needs confirmation.

\section{Expert opinion 3: Dr. Ory Wiesel}

Few studies have evaluated the prognostic impact of PD-L1 expression in NSCLC. However, the results of these studies vary; while some studies have demonstrated that PD-L1 expression correlated with poor clinical outcome, others demonstrated a favorable prognosis for NSCLC with PD-L1 expression. As PD-L1 expression and response to immunotherapy is inconsistent, to-date there is not enough data to guide treatment strategies based only on PD-L1 expression.

\section{Conclusions}

Adjuvant therapy is necessary in patients with initiallyevaluated advanced NSCLC. The exact duration or timing of adjuvant immunotherapy should be individually adapted to the current clinical situation and the drug-related adverse events. Chemotherapy plus $\mathrm{PD}(\mathrm{L}) 1$ inhibitors is recommended in adjuvant strategy for improving PFS and OS. Resuming immunotherapy is preferred once the patients recurs given the nice response in the preoperative setting. PD-L1 expression in relation to prognosis both in the adjuvant/neoadjuvant chemotherapy is still controversial, and needs confirmation.

\section{Acknowledgments}

Funding: None.

\section{Footnote}

Conflicts of Interest: The authors have no conflicts of interest to declare.

Ethical Statement: The authors are accountable for all aspects of the work in ensuring that questions related to the accuracy or integrity of any part of the work are appropriately investigated and resolved. Written informed consent was obtained from the patient for publication of this manuscript and any accompanying images.

Open Access Statement: This is an Open Access article distributed in accordance with the Creative Commons Attribution-NonCommercial-NoDerivs 4.0 International License (CC BY-NC-ND 4.0), which permits the noncommercial replication and distribution of the article with the strict proviso that no changes or edits are made and the original work is properly cited (including links to both the formal publication through the relevant DOI and the license). See: https://creativecommons.org/licenses/by-nc-nd/4.0/.

\section{References}

1. Gettinger S, Horn L, Jackman D, et al. Five-Year Follow- 
Up of Nivolumab in Previously Treated Advanced NonSmall-Cell Lung Cancer: Results From the CA209-003 Study. J Clin Oncol 2018;36:1675-84.

2. Herbst RS, Morgensztern D, Boshoff C. The biology and management of non-small cell lung cancer. Nature 2018;553:446.

3. NCCN Guidelines For Non-Small Cell Lung Cancer. National Comprehensive Cancer Network Non-Small Cell Lung Cancer (Version 52019). Available online: https://wwwnccnorg/professionals/physician_gls/pdf/ nsclpdf, accessed June 7, 2019.

4. Chen W, Zheng R, Baade PD, et al. Cancer statistics in China, 2015. CA Cancer J Clin 2016;66:115-32.

5. Planchard D, Popat S, Kerr K, et al. Metastatic non-small cell lung cancer: ESMO Clinical Practice Guidelines for diagnosis, treatment and follow-up. Ann Oncol 2019;30:863-70.

6. Liang H, Liu Z, Cai X, et al. PD-(L)1 inhibitors vs. chemotherapy vs. their combination in front-line treatment for NSCLC: An indirect comparison. Int J Cancer 2019;145:3011-21.

7. Antonia SJ, Villegas A, Daniel D, et al. Durvalumab after Chemoradiotherapy in Stage III Non-Small-Cell Lung Cancer. N Engl J Med 2017;377:1919-29.

8. Reck M, Rodríguez-Abreu D, Robinson AG, et al. Updated Analysis of KEYNOTE-024 Pembrolizumab Versus Platinum Based Chemotherapy for Advanced Non-Small-Cell Lung Cancer With PD-L1 Tumor Proportion Score of $50 \%$ or Greater. J Clin Oncol 2019;37:537-46.

9. Peng J, Hamanishi J, Matsumura N, et al. Chemotherapy Induces Programmed Cell Death-Ligand 1 Overexpression via the Nuclear Factor- $\kappa \mathrm{B}$ to Foster an Immunosuppressive Tumor Microenvironment in Ovarian Cancer. Cancer Research 2015;75:5034-45.

10. Qiao M, Jiang T, Ren S, et al. Combination Strategies on the Basis of Immune Checkpoint Inhibitors in Non-SmallCell Lung Cancer: Where Do We Stand? Clin Lung Cancer 2018;19:1-11.

11. Hegde PS, Karanikas V, Evers S. The Where, the

Cite this article as: Liang H, Deng $\mathrm{H}$, Liang W, Guo K, Gao Z, Wiesel O, Flores RM, Song K, Redwan B, Migliore M, Li S, Yin W, He J. Perioperative chemoimmunotherapy in a patient with stage IIIB non-small cell lung cancer. Ann Transl Med 2020;8(5):245. doi: 10.21037/atm.2020.01.118
When, and the How of Immune Monitoring for Cancer Immunotherapies in the Era of Checkpoint Inhibition. Clin Cancer Res 2016;22:1865-74.

12. Forde PM, Chaft JE, Smith KN, et al. Neoadjuvant PD-1 Blockade in Resectable Lung Cancer. N Engl J Med 2018;378:1976-86.

13. Zhang $\mathrm{P}, \mathrm{Ma} \mathrm{Y}, \mathrm{Lv} \mathrm{C}$, et al. Upregulation of programmed cell death ligand 1 promotes resistance response in nonsmall-cell lung cancer patients treated with neo-adjuvant chemotherapy. Cancer Sci 2016;107:1563-71.

14. Pirker R, Filipits M. Adjuvant Therapy in Patients With Completely Resected Non-small-cell Lung Cancer: Current Status and Perspectives. Clin Lung Cancer 2019;20:1-6.

15. Pignon JP, Tribodet H, Scagliotti GV, et al. Lung adjuvant cisplatin evaluation: a pooled analysis by the LACE Collaborative Group. J Clin Oncol 2008;26:3552-9.

16. Strauss GM, Herndon JE 2nd, Maddaus MA, et al. Adjuvant paclitaxel plus carboplatin compared with observation in stage IB non-small-cell lung cancer: CALGB 9633 with the Cancer and Leukemia Group B, Radiation Therapy Oncology Group, and North Central Cancer Treatment Group Study Groups. J Clin Oncol 2008;26:5043-51.

17. Owen D, Chaft JE. Immunotherapy in surgically resectable non-small cell lung cancer. J Thorac Dis 2018;10:S404-S411.

18. Gao G, Qiao M, Liu H, et al. Impact of clinicopathological features on the efficacy of immune checkpoint inhibitors plus conventional treatment in patients with advanced lung cancer. J Thorac Dis 2019;11:3794-807.

19. Lacour M, Hiltbrunner S, Lee SY, et al. Adjuvant Chemotherapy Increases Programmed Death-Ligand 1 (PD-L1) Expression in Non-small Cell Lung Cancer Recurrence. Clin Lung Cancer 2019;20:391-6.

20. Owada-Ozaki Y, Muto S, Takagi H, et al. Prognostic Impact of Tumor Mutation Burden in Patients With Completely Resected Non-Small Cell Lung Cancer: Brief Report. J Thorac Oncol 2018;13:1217-21. 